\title{
Insulinoma: Reversal of brain magnetic resonance imaging changes following resection
}

\author{
Rajiv Pakhetra, Gagan Priya, Viveka P. Jyotsna, Ashu Seith ${ }^{1}$, Ariachery C. Ammini \\ Departments of Endocrinology and Metabolism, ${ }^{1}$ Radiodiagnosis, All India Institute of Medical Sciences, Ansari Nagar, New Delhi - 110029 , \\ India
}

\begin{abstract}
Insulinoma presents with myriad manifestations and severe neurological deficit may develop due to delay in diagnosis. We report a lady who presented with Glasgow coma scale of E1 M2 V1, which did not improve after correction of hypoglycemia. There was complete reversal of neurological deficit and brain magnetic resonance imaging changes of hypoglycemia on follow-up after resection of pancreatic insulinoma.

This is the first report which shows reversal of hypoglycemic changes in MRI after resection of insulinoma. Insulinoma, pre and post surgery provides a model for study of the effect of hypoglycemia and its improvement after euglycemia.
\end{abstract}

Key words: Brain magnetic resonance imaging changes, hypoglycemic coma, insulinoma, recovery, reversal

Insulinoma can present with disorders of cognition, consciousness, epilepsy, psychosis, dementia and neuropathy.$^{[1-5]}$ Misdiagnosis and delay in treatment are common and may lead to prolonged hypoglycemia with permanent neurological deficit or fatal coma. ${ }^{[3]}$

Diagnosis depends on inappropriately high insulin levels in presence of hypoglycemia which could occur spontaneously or during prolonged fast. ${ }^{[5]}$ Surgical removal of the tumor corrects the hyperinsulinemia and restores euglycemia.

But there is little literature on whether neurological deficit due to hypoglycemia-induced cerebral damage, which persists after correction of hypoglycemia, improves after surgical removal of the tumor. ${ }^{[3,6,7]}$ We report a case of a 27-year-old lady with insulinoma who presented with significant neurological deficit, which persisted despite normalization of blood glucose levels. Magnetic resonance imaging (MRI) brain revealed changes due to hypoglycemia. After the insulinoma was resected, there was a gradual and significant neurological improvement and MRI changes reverted. This is the first report which shows reversal of hypoglycemic changes in MRI after resection of insulinoma.

\section{Case Report}

A 27 years of age, was in good health until six months prior to her hospitalization. During the past six months, she had often been found in an unresponsive state in the morning, recovering spontaneously towards afternoon. There was no history of sweating or other autonomic symptoms preceding the episodes of alteration of consciousness. She had no history of increased appetite or weight gain. She was started on anti-epileptic medication (phenytoin $300 \mathrm{mg} /$ day) for these complaints. Electroencephalogram and magnetic resonance imaging brain were found to be normal. Due to recurring episodes of altered consciousness, anti-epileptic treatment was modified several times (carbamazepine $600 \mathrm{mg} /$ day and valproic acid, $2000 \mathrm{mg} /$ day). Her symptomatology, however, persisted.

She was found unconscious one morning and taken to a nursing home, where her blood glucose was documented to be $31 \mathrm{mg} / \mathrm{dl}$. Dextrose infusion was started but sensorium did not improve. She was then referred to our institute. At admission, she was unconscious with a Glasgow coma score of $E_{1}$ $\mathrm{M}_{2} \mathrm{~V}_{1}$ and required continuous dextrose infusion to maintain normoglycemia. Serum insulin was found to be $14.3 \mu \mathrm{U} / \mathrm{ml}$ with corresponding blood glucose of $50 \mathrm{mg} / \mathrm{dl}$. Hemogram, liver and renal functions were normal. Serum sodium was 140 meq/l (normal 130-149 $\mathrm{meq} / \mathrm{L}$ ) and serum potassium was $4 \mathrm{meq} / \mathrm{l}$ (normal 3.5 to $5 \mathrm{meq} / \mathrm{l})$. The HbA1c was $3.8 \%$; serum T4, TSH and basal cortisol were normal. Magnetic resonance imaging of the brain was done on the fifth day of admission. Diffusion-weighted brain MRI sequences showed a hyperintense signal in the genu of the left internal capsule extending into the posterior limb and thalamus. A similar small signal abnormality was seen on the right side [Figures 1A, B].

She had recurrent episodes of hypoglycemia during the hospital stay which required almost continuous intravenous glucose infusion. 


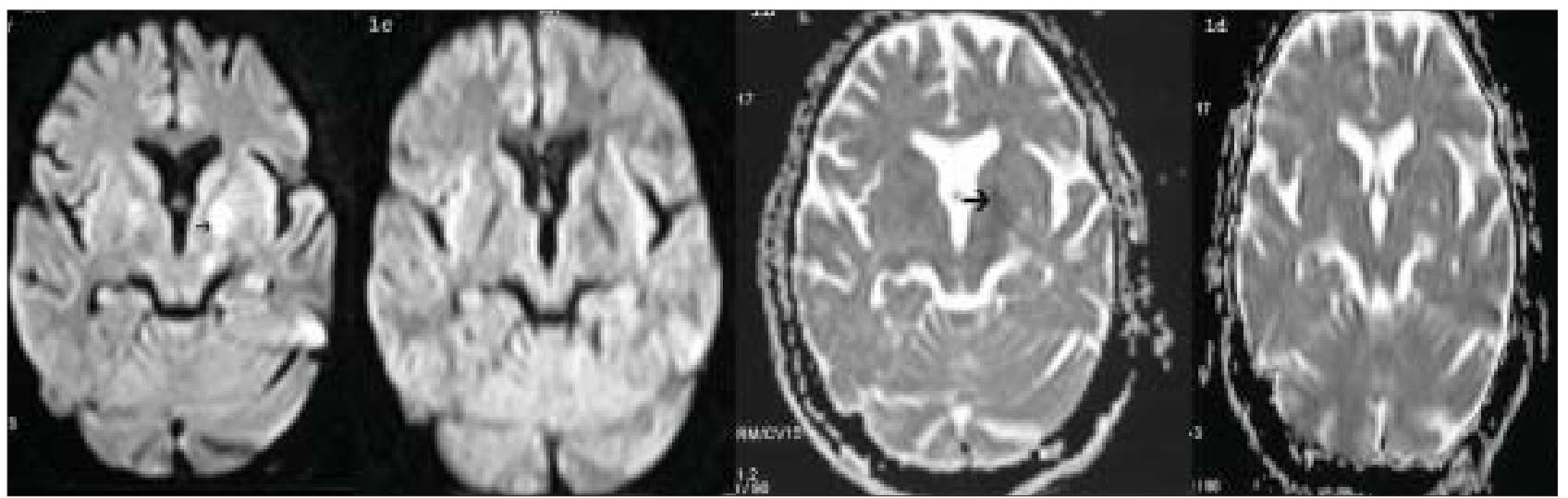

Figure 1: (A) Diffusion-weighted MRI sequences showing hyperintensity in the genu of the left internal capsule (arrow) extending into the posterior limb and thalamus. A similar but smaller signal abnormality is seen on the right side. (B) The corresponding apparent diffusion coefficient map showing hypointensity (arrow) in the same region-restricted diffusion. (C) Resolution of signal abnormalities on diffusionweighted MRI sequences 16 months later. (D) Resolution of signal abnormalities on corresponding ADC map 16 months later

A dual-phase computed tomography scan of the abdomen revealed a 3 -cm well-defined, homogenous mass with increased vascularity in the tail of the pancreas.

During the course of the preoperative evaluation, she developed a central venous line infection and surgery had to be delayed. Diazoxide was started at a dose of $150 \mathrm{mg} /$ day and increased up to $300 \mathrm{mg} /$ day, following which her requirement of intravenous dextrose to maintain normoglycemia decreased significantly. She regained consciousness with spontaneous eye opening within two weeks but still remained disoriented. She could make out sounds but words spoken were incoherent and she was unable to recognize anyone. She had significant weakness of all four limbs (power $2 / 5$ ) with poorly elicitable deep tendon reflexes.

Considering her general condition and neurological damage there was speculation as to how much her neurological condition would improve after surgical removal of insulinoma.

She underwent distal pancreatectomy and splenectomy. Histopathological examination of the excised tissue revealed a well-differentiated pancreatic endocrine tumır. She had transient hyperglycemia in the immediate postoperative period, necessitating insulin therapy for about 10 days. At discharge, she was euglycemic and did not require insulin or oral hypoglycemic drugs. She was alert and oriented. She was able to speak full sentences but had significant dysarthria. She could sit without support but required assistance to stand and perform activities of daily living.

Three months later, she was ambulant and able to perform basic daily chores. She had some residual weakness in the lower limbs and dysarthria. Fourteen months after surgery, residual weakness in her lower limbs and dysarthria had disappeared. The MRI brain done at 16 months revealed complete reversal of the signal abnormalities on diffusionweighted images [Figures 1C, D].

\section{Discussion}

Neurological involvement in the form of recurrent seizures, cognitive dysfunction and loss of consciousness are common in insulinoma. ${ }^{[1-7]}$ The risk for prolonged or permanent neurological injury is likely related to the severity and duration of hypoglycemia. In situations of severe prolonged hypoglycemia, neurological deficits may persist for days to weeks despite correction of hypoglycemia. Neurological deficits are more commonly reported in patients with diabetes ${ }^{[6,7]}$ who are hypoglycemic than in non-diabetic persons with a hypoglycemic disorder such as insulinoma. ${ }^{[3]}$

Hypoglycemia injures the middle layers of the cerebral cortex and hippocampus and spares the brainstem and spinal cord. ${ }^{[8-10]}$ Central pontine myelinosis has been reported in dyselectrolytemia, diabetics, anorectics and sudden changes in serum osmolality. It characteristically affects the pons as well as certain extrapontine sites such as the internal capsule, basal ganglia, cerebellum and cerebrum. In this case there was no record which showed that the patient ever had dyselectrolytemia or sudden correction of dyselectrolytemia. Also, the MRI showed lesion only in the extrapontine area without the pons being affected. Though, there are reports of extrapontine myelinolysis accompanying central pontine myelinolysis occurring in the setting of diabetic ketoacidosis, hypoglycemic coma, and anorexia nervosa, there are no reports of isolated extrapontine myelinolysis in adults with these conditions. Therefore it was not considered a case of central pontine myelinolysis.

Daggett et al., ${ }^{[3]}$ in their series of 30 patients, reported three patients between 30 to 34 years of age who had significant neurological deficits which persisted after correction of hypoglycemia but recovered significantly on long-term follow-up after tumor removal. In the first patient, the only major disability two years 
following tumor removal was palatal paralysis. The second patient had been unconscious for two days and thereafter was severely obtunded. Following removal of insulinoma, he improved gradually but was left with a marked intellectual impairment. Six months later, his wife reported that he had returned completely to normal. The third patient developed decerebrate rigidity after having been unconscious for 10 days. Neurological features then showed marked fluctuation despite maintaining normoglycemia. They included cerebellar ataxia, expressive dysphasia and left homonymous hemianopia. She improved only very slowly after removal of her insulinoma. During recovery, a peripheral neuropathy affecting intrinsic muscles of the hands was found which resolved over a two-year period. She was eventually left only with residual spastic dysarthria and cerebellar ataxia.

A few other reports describe reversal of dementia ${ }^{[4]}$ and neuropsychiatric symptoms ${ }^{[6,7]}$ following surgical treatment of insulinoma. To our knowledge, this is the first report which shows reversal of hypoglycemic changes in MRI after surgical removal of insulinoma. Insulinoma pre and post surgery can in future provide a model for the study of the effects of hypoglycemia and how it improves when euglycemia is restored. Even though patients might have severe hypoglycemic brain damage, it may still be worthwhile to resect the insulinoma, as there is good chance of recovery.

\section{References}

1. Best JD, Chisholm DJ, Alford FP. Insulinoma: Poor recognition of clinical features is the major problem in diagnosis. Med J Aust 1978;2:1-5.

2. Graves TD, Gandhi S, Smith SJ, Sisodiya SM, Conway GS. Misdiagnosis of seizures: Insulinoma presenting as adult onset seizure disorder. J Neurol Neurosurg Psychiatry 2004;75:1091-2.

3. Daggett P, Nabarro J. Neurological aspects of insulinomas. Postgrad Med J 1984;60:577-81.

4. Jyotsna VP, Rangel N, Pal S, Seith A, Sahni P, Ammini AC. Insulinoma: Diagnosis and surgical treatment: Retrospective analysis of 31 cases. Indian J Gastroenterol 2006;25:244-7.

5. Queiroz Almeida M, Machado MC, Correa-Giannella ML, GiannellaNeto D, Alberqaria, Pereira MA. Endogenous hyperinsulinemic hypoglycemia: Diagnostic strategies, predictive features of malignancy and long term survival. J Endocrinol Invest 2006;29:679-87.

6. Vig S, Lewis M, Foster KJ, Stacey-Clear A. Lessons to be learnt: A case study approach insulinoma presenting as a change in personality. J R Soc Health 2001;121:56-61.

7. Olsen DB, Abraham JH. Neuropsychiatric disorders in insulinoma. Ugeskr Laeger 1999;161:1420-1.

8. Aoki T, Sato T, Hasegawa K, Ishizaki R, Saiki M. Reversible hyperintensity lesion on diffusion- weighted MRI in hypoglycemic coma. Neurology 2004;63:392-3.

9. Finelli F. Diffusion weighted MR in hypoglycemic coma. Neurology 2001;57:933-5.

10. Honma R, Saitoh T, Kameda W, Ohizumi T, Yamaguchi H, Ohnuma H, \&al. Hyperintense hippocampus on diffusion-weighted MR images in a case of insulinoma with persistent memory disturbance. Nippon Naika Gakkai Zasshi 2003;92:137-9.

Accepted on 05-03-2008

Source of Support: Nil, Conflict of Interest: None declared. 